\title{
The precise NNLO PDFs from the combined fit to the DIS and Drell-Yan data
}

\author{
Sergey Alekhin*† \\ Institute for High Energy Physics, Protvino, 142281 Russia \\ E-mail: alekhin@ihep.ru
}

\begin{abstract}
We determine parton distribution functions (PDFs) from the fit to deep-inelastic-scattering (DIS) data for the proton and deuteron targets and the fixed-target Drell-Yan (DY) data by the E605 and E866 experiments at Fermilab. Full set of the next-to-next-to-leading (NNLO) QCD corrections to the evolution kernel and to the coefficient functions is taken into account. Thanks to the DY data, the experimental error in sea quark distributions is less than $20 \%$ up to $x \sim 0.7$, while the error due to variations of the factorization and renormalization scales is less than or comparable to the experimental one.
\end{abstract}

International Europhysics Conference on High Energy Physics

July 21st - 27th 2005

Lisboa, Portugal

*Speaker.

${ }^{\dagger}$ Supported by the RFBR grant 03-02-17177 and by the Russian Ministry of Science grant NSh 1695.2003.2. 
Determination of parton distribution functions (PDFs) in nucleon with next-to-next-to-leading order (NNLO) accuracy in QCD is important for testing perturbative stability of theoretical predictions for scattering cross sections in hadron collisions [1]. The NNLO coefficient functions to deep-inelastic-scattering (DIS) were computed in [2]. Thanks to recent calculations of the NNLO evolution kernels [3] and the NNLO corrections to the coefficient functions of the Drell-Yan process [4], self-consistent determination of the NNLO PDFs from a fit to combined DIS and DY data is possible. The purpose of this paper is to present the results of such a fit.

In the analysis, we use charged-lepton DIS data [5] together with the results of Fermilab highstatistics experiments on fixed-target muon pair production. The latter provide data for the isoscalar target [6] and the ratio of cross sections for the deuteron and proton targets [7] with the accuracy better than $20 \%$ at $x \lesssim 0.6$. It turns out that these data are indispensable for constraining sea quark distributions in the nucleon. This happens because for $x \lesssim 0.6$ the DIS data constrain valence quark distributions with the precision of about $1 \%$ [8]; therefore, the combined fit enables determination of the sea distributions with accuracy comparable to the precision of the DY data.

In order to suppress sensitivity to higher twist (HT) terms, the DIS data with $Q^{2}<2.5 \mathrm{GeV}^{2}$ and $W<1.8 \mathrm{GeV}$ are not used in the fit. Nevertheless, HT terms are taken into account through the target mass correction and additional dynamical HT terms, parameterized in a model-independent way using cubic splines. The lowest value of $Q^{2}$ for the DY data used in the fit is about $25 \mathrm{GeV}^{2}$; therefore, the HT terms are inessential for these data.

For the deuteron DIS data we apply corrections for nuclear effects, which include Fermi motion, shadowing, and off-shellness of the nucleon [9]. Since we have sufficient confidence in these corrections, we do not apply a cut on $x$ and include in the fit the DIS data points up to $x=0.9$, the largest value available in the set of Ref.[5]. The nuclear corrections to DY cross sections are not taken into account since the DY data used in the fit are concentrated in the region where nuclear effects are small. Heavy-quark contributions to the DIS cross section are taken into account within the massive factorization scheme using calculations of Ref. [10]. For the kinematics of the DY data used in the fit, the heavy-quark contribution is unimportant and we did not consider it.

We choose $Q=3 \mathrm{GeV}$ as the initial value for QCD scale evolution. The PDFs at that scale are parameterized as follows

$$
x p_{i}(x, Q=3 \mathrm{GeV})=N_{i} x^{a_{i}}(1-x)^{b_{i}} x^{\left(\gamma_{1} x+\gamma_{2} x^{2}+\cdots\right)} .
$$

Since the DIS and DY data constrain the strange quark sea distribution poorly, we use the dimuon data by CCFR collaboration [11] to fix it. The normalization factors $N_{i}$ for the valence quark and gluon distributions are calculated from other parameters in Eq.(1) using the fermion number and momentum conservation sum rules, respectively. For the non-strange sea distributions, they are fitted to the data together with $a_{i}, b_{i}$, and $\gamma_{i}$. Coefficients $\gamma_{i}$ give us sufficient flexibility to arrange for appropriate shapes of distribution functions; they are consequently added to Eq.(1) until statistically significant decrease of $\chi^{2}$ is achieved. It turns out that for the valence quark distributions we have to keep $\gamma_{1,2}$ and for the sea and gluon distributions keeping just $\gamma_{1}$ is sufficient.

The non-strange sea PDFs obtained in the fit to combined DIS and DY data with their $1 \sigma$ error bands determined from statistical and systematic uncertainties in the experimental data are given in Fig.1. We observe significant improvement in the precision of the sea distributions compared to the analysis based on the DIS data only. The achieved precision of the total sea distribution is 


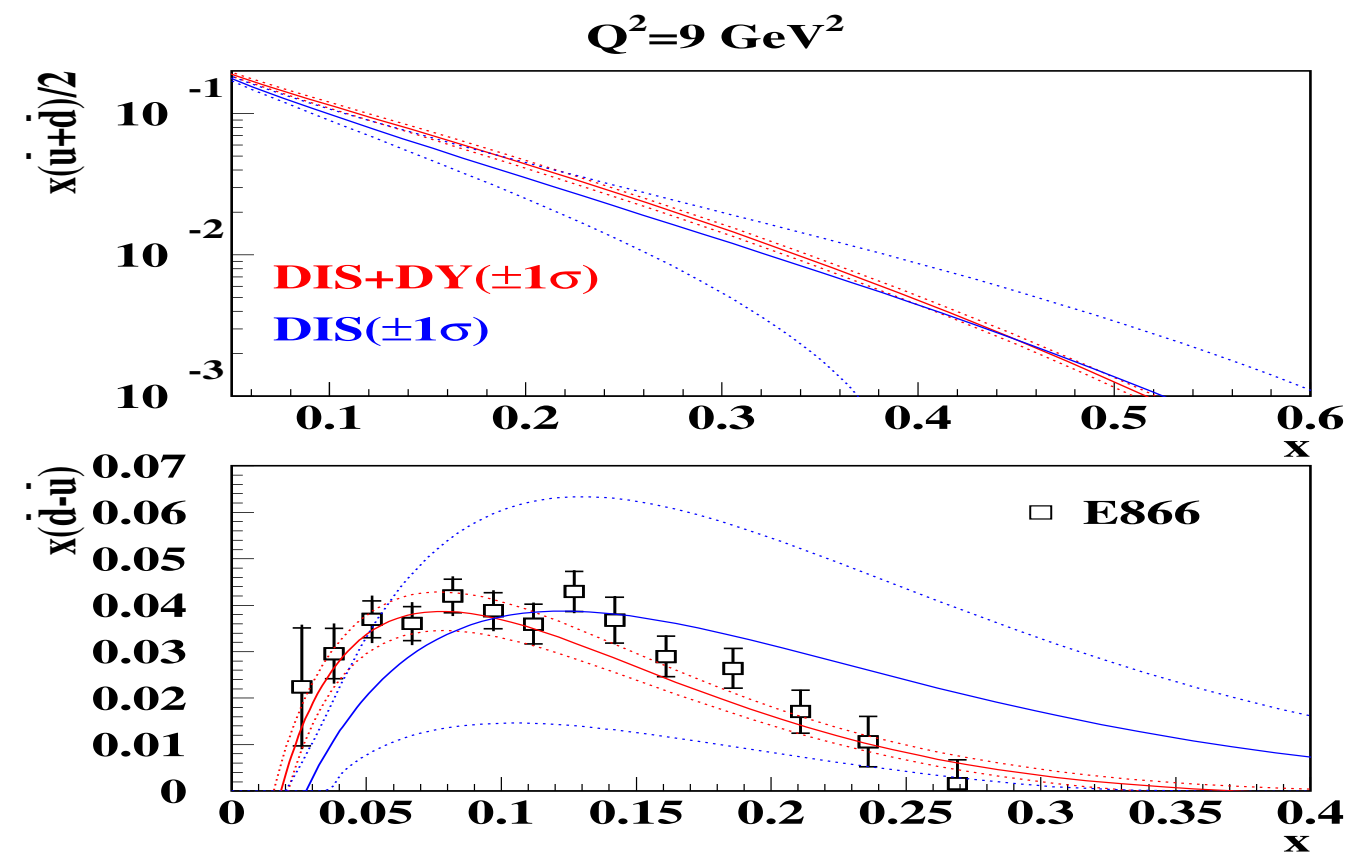

Figure 1: The central values (solid) and the $1 \sigma$ bands for the PDFs extracted from the combined DIS and DY data (red) compared to one based on the DIS data only (blue). The points at lower panel give results of the analysis of Ref. [7].

in good agreement with the expectation based on relative uncertainties of the DIS and DY data used in the fit. The value of $\chi^{2} / \mathrm{NDP}$ obtained in the fit is 1.1 and the spread of $\chi^{2} / \mathrm{NDP}$ over separate experiments is not dramatic; its largest value is about 1.4. We rescaled the errors in data for experiments with $\chi^{2} / \mathrm{NDP}>1$ in order to bring $\chi^{2} / \mathrm{NDP}$ for these experiments to 1 and found that an overall impact of this rescaling on the PDFs errors is marginal. This proves sufficient statistical consistency of the analyzed data and disfavors criterion $\Delta \chi^{2}=100$ used by the CTEQ collaboration in their fit [12]. Given good consistency of the data, we can estimate the error in PDFs using statistically rigorous criterion $\Delta \chi^{2}=1$. As the result, the errors in sea distributions obtained in our analysis are much smaller than uncertainties given by the CTEQ collaboration (see Fig.2). Finally, we note that PDFs obtained in our analysis are rather stable against variations of the factorization and renormalization scales in the DY cross section, Fig.2. Variation of these scales within the range $M_{\mu^{+} \mu^{-}} / 2<\mu<2 M_{\mu^{+} \mu^{-}}$leads to changes in PDFs comparable to their uncertainties due to errors in the data.

\section{References}

[1] A. Cafarella, C. Coriano, M. Guzzi and J. Smith, hep-ph/0510179.

[2] E. B. Zijlstra and W. L. van Neerven, Nucl. Phys. B 383 (1992) 525;

D. I. Kazakov and A. V. Kotikov, Phys. Lett. B 291 (1992) 171. 

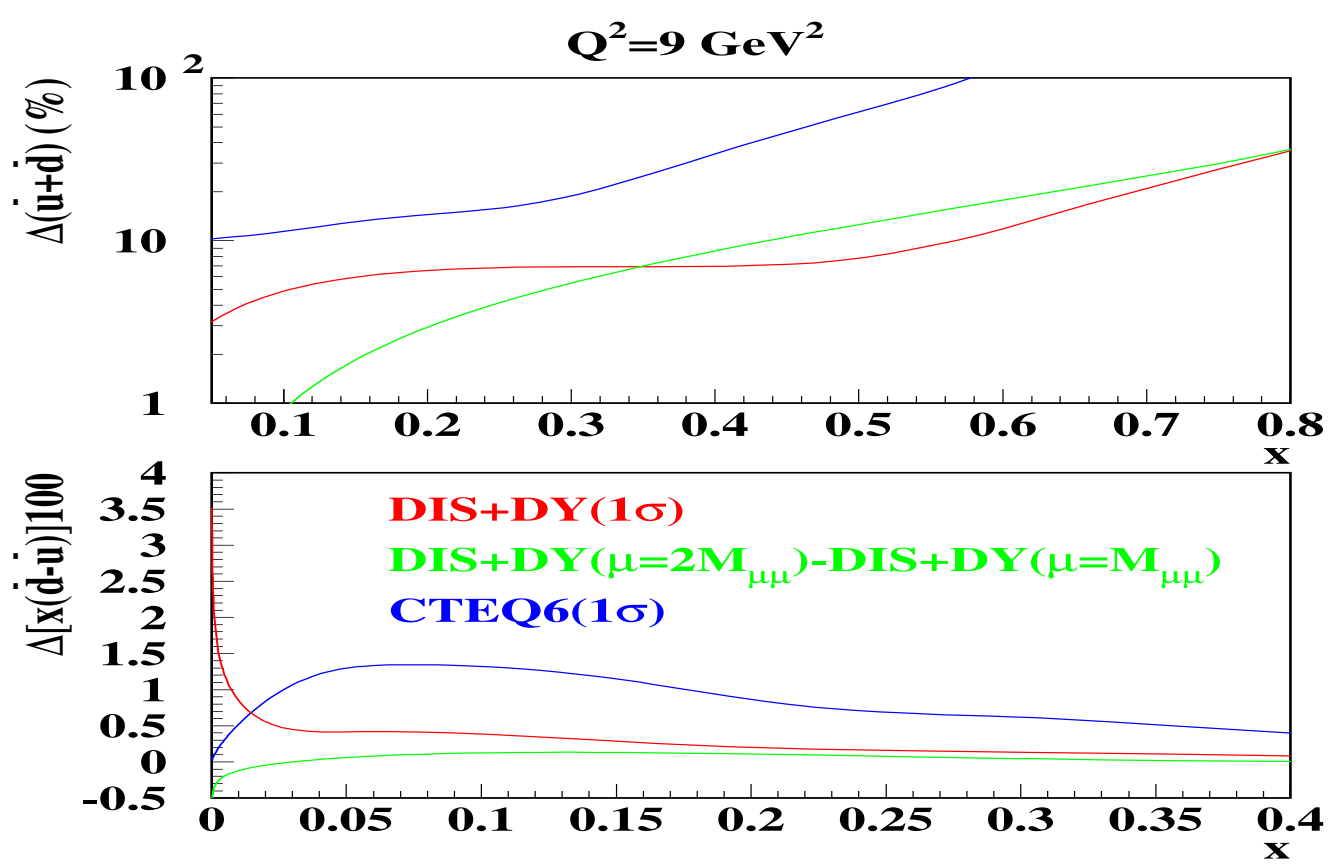

Figure 2: The $1 \sigma$ errors in PDFs due to the uncertainties in data obtained in this analysis (red) compared to one obtained by the CTEQ collaboration (blue) and to the uncertainties due to variation of the renormalization/factorization scale (green).

[3] S. Moch, J. A. M. Vermaseren and A. Vogt, Nucl. Phys. B 688 (2004) 101 [hep-ph/ 0403192 ]; A. Vogt, S. Moch and J. A. M. Vermaseren, Nucl. Phys. B 691 (2004) 129 [hep-ph / 0404111 ].

[4] C. Anastasiou, L. J. Dixon, K. Melnikov and F. Petriello, Phys. Rev. Lett. 91 (2003) 182002 [hep-ph/0306192].

[5] L. W. Whitlow, E. M. Riordan, S. Dasu, S. Rock and A. Bodek, Phys. Lett. B 282 (1992) 475; A. C. Benvenuti et al. [BCDMS Collaboration], Phys. Lett. B 223 (1989) 485; A. C. Benvenuti et al. [BCDMS Collaboration], Phys. Lett. B 237 (1990) 592; M. Arneodo et al. [New Muon Collaboration], Nucl. Phys. B 483 (1997) 3 [hep-ph/9610231]; C. Adloff et al. [H1 Collaboration], Eur. Phys. J. C 21 (2001) 33 [hep-ex/ 0012053 ]; S. Chekanov et al. [ZEUS Collaboration], Eur. Phys. J. C 21 (2001) 443 [hep-ex/ 0105090 ].

[6] G. Moreno et al. [FNAL E605 Collaboration], Phys. Rev. D 43 (1991) 2815.

[7] R. S. Towell et al. [FNAL E866/NuSea Collaboration], Phys. Rev. D 64 (2001) 052002 [hep-ex/0103030].

[8] S. Alekhin, JETP Lett. 82 (2005) 628 [hep-ph/ 0508248$].$

[9] S. A. Kulagin and R. Petti, hep-ph/0412425.

[10] E. Laenen, S. Riemersma, J. Smith and W. L. van Neerven, Nucl. Phys. B 392 (1993) 229.

[11] A. O. Bazarko et al. [CCFR Collaboration], Z. Phys. C 65 (1995) 189 [hep-ex/9406007].

[12] Pumplin, J. et al., JHEP 07 (2002) 012. 